\title{
URBAN SCENE CLASSIFICATION USING FEATURES EXTRACTED FROM PHOTOGRAMMETRIC POINT CLOUDS ACQUIRED BY UAV
}

\author{
G. G. Pessoa ${ }^{1,}$, R. C. Santos ${ }^{1}$, A. C. Carrilho ${ }^{1}$, M. Galo ${ }^{1,2}$, A. Amorim ${ }^{1,2}$ \\ ${ }^{1}$ São Paulo State University - UNESP, Graduate Program in Cartographic Sciences, \\ Presidente Prudente, São Paulo, Brazil - guilhermegpessoa@hotmail.com, renato_cstos@hotmail.com, carrilho.acc@gmail.com \\ ${ }^{2}$ São Paulo State University - UNESP, Dept. of Cartography, \\ Presidente Prudente, São Paulo, Brazil - mauricio.galo@unesp.br, a.amorim@unesp.br
}

Commission II, WG III/3

KEY WORDS: Photogrammetric Point Cloud Classification, UAV, Urban Scene Classification

\begin{abstract}
:
Images and LiDAR point clouds are the two major data sources used by the photogrammetry and remote sensing community. Although different, the synergy between these two data sources has motivated exploration of the potential for combining data in various applications, especially for classification and extraction of information in urban environments. Despite the efforts of the scientific community, integrating LiDAR data and images remains a challenging task. For this reason, the development of Unmanned Aerial Vehicles (UAVs) along with the integration and synchronization of positioning receivers, inertial systems and off-the-shelf imaging sensors has enabled the exploitation of the high-density photogrammetric point cloud (PPC) as an alternative, obviating the need to integrate LiDAR and optical images. This study therefore aims to compare the results of PPC classification in urban scenes considering radiometric-only, geometric-only and combined radiometric and geometric data applied to the Random Forest algorithm. For this study the following classes were considered: buildings, asphalt, trees, grass, bare soil, sidewalks and power lines, which encompass the most common objects in urban scenes. The classification procedure was performed considering radiometric features (Green band, Red band, NIR band, NDVI and Saturation) and geometric features (Height - nDSM, Linearity, Planarity, Scatter, Anisotropy, Omnivariance and Eigenentropy). The quantitative analyses were performed by means of the classification error matrix using the following metrics: overall accuracy, recall and precision. The quantitative analyses present overall accuracy of $0.80,0.74$ and 0.98 for classification considering radiometric, geometric and both data combined, respectively.
\end{abstract}

\section{INTRODUCTION}

Urban scene complexity is one of the main factors that makes it difficult to discriminate objects, mainly by automatic classification methods. In addition, the lack of information due do the occurrence of occlusions and the characteristics of the equipment are further aspects hampering the classification process (Sohn \& Dowman, 2007). In past decades aerial images and LiDAR point clouds were the two main data sources used in urban environment analysis, such as Land Use and Land Cover (LULC) mapping (Zhang \& Lin, 2017; Rau et al., 2015; Ali-Sisto \& Packalen, 2017), building modelling (Haala \& Kada, 2010), etc.

The synergy between these two main data sources has motivated several studies aiming at the integration of images and LiDAR point clouds in order to explore the complementarity of radiometric and geometric information (Hermosilla et al., 2011; Buján et al., 2012; Malpica et al., 2013). Recent advances in the development of Unmanned Aircraft Vehicle (UAV), positioning sensors and inertial sensors concomitant with the development of algorithms for image processing and computer vision have enabled the exploitation of the potential of information extraction from photogrammetric point clouds as an alternative to the integration of LiDAR data and images (Rau et al., 2015; and Ali-Sisto \& Packalen, 2017; Nex \& Remondino, 2014).
Although the development of UAV has enabled major advances in data acquisition, the payload capacity sets limitations to the amount and quality of the embedded sensors (Nex \& Remondino, 2014 and Colomina \& Molina, 2014). The availability of radiometric information can vary as a function of the sensors embedded, ranging from few bands to many in the case of hyperspectral sensors, as can be seen in Aasen et al. (2015) and Näsi et al. (2018). In the case of point clouds resulting from the photogrammetric processing of images obtained by UAV, the payload limitations directly affect the availability of radiometric information since the sensors used usually allow the acquisition of a reduced number of bands. In addition to the radiometric data limitations, another problem is related to positional accuracy of the point cloud and the relatively high frequency of spurious points if compared to LiDAR data, which directly affects the quality of extracted objects. In these cases, exploitation of the complementarity of radiometric and geometric information is even more important.

In this context, this study explores the PPC classification applying radiometric and geometric features extracted directly over the PPC from the RF classifier. The following classes were considered: buildings, asphalt, trees, grass, bare soil, sidewalks and power lines, which encompass the most common objects in urban scenes. Three experiments were carried out, one considering only radiometric features, one considering just geometric features and the third considering both radiometric and geometric features. Quantitative analyses were performed

\footnotetext{
${ }^{*}$ Corresponding author
} 
by means of the classification error matrix using the following metrics: overall accuracy, recall and precision.

\section{METHOD}

Figure 1 presents a simplified flowchart of the proposed method. Although the flight to acquire the images and photogrammetric processing for the PPC generation were performed (Section 3.1), the flowchart starts from the available point cloud. In general, the proposed method can be divided into three main steps: point cloud pre-processing, feature extraction and Random Forest classifier training and application.

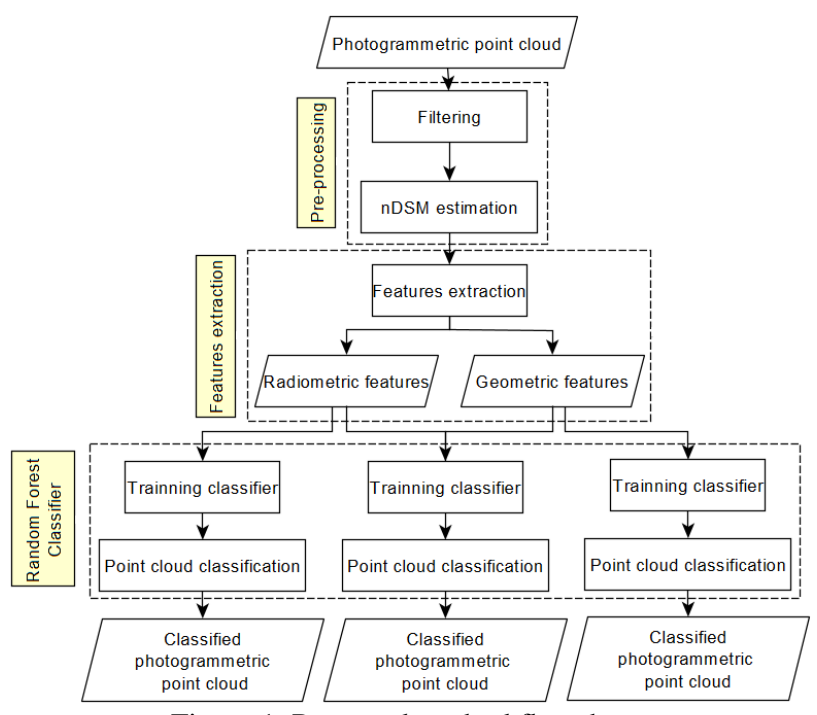

Figure 1. Proposed method flowchart.

\subsection{Pre-processing}

In this work, the pre-processing step comprises applying filtering techniques to extract Digital Terrain Model (DTM), and then normalized Digital Surface Model (nDSM) estimation from the DTM and Digital Surface Model (DSM) subtraction. According to Li et al. (2004), a DSM may contain information on various elements present in a scene, including vegetation, buildings and other objects while DTM consists only of the points that are directly on the ground. The nDSM represents the absolute elevation of the features above terrain level.

Filtering techniques are designed to detect and/or eliminate non-terrain points. This process can be performed directly on the original cloud (Pfeifer et al., 1998; Axelsson, 1999; Sithole \& Vosselman, 2001) or considering a point cloud rasterization process in order to apply image processing techniques. In addition, the filtering algorithms can be classified in seven groups (Han et al. 2017): Statistically-based filtering techniques; Neighborhood-based filtering techniques; Projection-based filtering approaches; Signal-processing based method; Partial Differential Equations-based filtering technique; Hybrid filtering techniques and others.

The rapidlasso LAStools toolkit was applied to carry out the filtering process. This toolkit was developed by Martin Isenburg, and is being constantly modernized. It provides filtering and DTM extraction tools, which use a variant of the "Progressive TIN Densification" filtering algorithm proposed by Axelsson (2000).
Progressive TIN Densification (PTD) is a classic filtering method widely applied in both the scientific and commercial communities (Zhang \& Lin, 2013). PTD consists of an iterative approach and works directly on the original point cloud. PDT is basically composed of two steps: initial TIN determination from seed points and the iterative densification of the initial TIN. In the first step, the set of points is subdivided into a regular grid of pre-established size, taking into account the size of elevated objects present in the scene, and then the minimum point is selected from each grid cell as the seed point in order to construct the initial TIN. In the progressive densification step, one point could be added to each TIN facet at each iteration if it meets the criteria on two parameters: the distance to the facet and the angles to the nodes. The PDT progressive densification step ends with the iteration at which no points are added to the TIN facets.

\subsection{Feature extraction}

In the context of automated classification methods, feature extraction is a fundamental step, especially for machine learning approaches (Meyer-Baese, 2014). The feature extraction step can be carried out in order to calculate metrics from the input data as well as the available features. Thus, a feature may be defined as a function of one or more quantifiable property (ie, color, shape, frequency, texture, etc). Two set of features are considered in this work: radiometric and geometric.

The radiometric features considered consist of the red (R), green $(\mathrm{G})$, and near-infrared (NIR) bands, captured by the sensor, the Normalized Difference Vegetation Index (NDVI), and the minimum (R, G, NIR), respectively $\mathrm{Eq}(\mathrm{s}) .1$ and 2. The motivation to include the feature based on the minimum (R, G, NIR) is relate to its capability to discriminate dark objects.

$$
\begin{gathered}
N D V I=\frac{N I R-R}{N I R+R} \\
m=\operatorname{minimum}(R, G, N I R)
\end{gathered}
$$

The geometric feature extraction was performed taking a local neighborhood into account. Considering that the neighborhood size has influence on the features, it is appropriate to use a methodology enabling selection of an "optimal neighborhood" (Demantké et al., 2011; Weinmann et al., 2015; Santos and Galo, 2018). The 'optimal neighborhood' may be selected using the entropy concept. In this work, an optimal neighborhood was considered for each point in the cloud within the boundaries of a minimum of 10 points and a maximum of 100 points in the neighborhood, as suggested by Weinmann et al. (2015). After the optimal neighborhood definition, the following geometric features are estimated (Weinmann et al., 2013, 2015; Blomley et al. 2014): Linearity $\left(\boldsymbol{L}_{\lambda}\right)$, Planarity $\left(\boldsymbol{P}_{\lambda}\right)$, Scatter (or Sphericity) $\left(\boldsymbol{S}_{\boldsymbol{\lambda}}\right)$, Anisotropy $\left(\boldsymbol{A}_{\boldsymbol{\lambda}}\right)$, Omnivariace $\left(\boldsymbol{O}_{\lambda}\right)$ and Eigenentropy $\left(\boldsymbol{E}_{\lambda}\right)$, as can be seen in $\mathrm{Eq}(\mathrm{s}) .3-8$ respectively. In addition to these, the height derived from the pre-processing step was also considered.

$$
\begin{aligned}
L_{\lambda} & =\frac{\lambda_{1}-\lambda_{2}}{\lambda_{1}} \\
P_{\lambda} & =\frac{\lambda_{2}-\lambda_{3}}{\lambda_{1}}
\end{aligned}
$$




$$
\begin{gathered}
\boldsymbol{S}_{\lambda}=\frac{\lambda_{3}}{\lambda_{1}} \\
\boldsymbol{A}_{\lambda}=\frac{\lambda_{1}-\lambda_{3}}{\lambda_{1}} \\
\boldsymbol{O}_{\lambda}=\sqrt[3]{\lambda_{1} * \lambda_{2} * \lambda_{3}} \\
\boldsymbol{E}_{\lambda}=-\sum_{i=1}^{3} \lambda_{i} * \ln \left(\lambda_{i}\right)
\end{gathered}
$$

where $\lambda_{1}, \lambda_{2}$, and $\lambda_{3}$, being $\lambda_{1}>\lambda_{2}>\lambda_{3}>0$, correspond to normalized eigenvalues computed from the $3 \mathrm{D}$ matrix. The matrix is determined considering the $\mathrm{X}, \mathrm{Y}, \mathrm{Z}$ coordinates of points inside the neighborhood.

\subsection{Random Forest classifier}

The radiometric and geometric features extracted were treated as input for the classifier to generate the classification results. At this point, several different classifiers could be considered. According to Breiman (2001), the Random Forest classifier has several advantages: it is robust to noise, fast processing compared to bagging and boosting refinement, and presents estimates of internal errors and the importance of each attribute in the classification process.

The RF is an ensemble learning method that uses a combination of several decision trees. The technique used to build the RF is a combination of the Bagging, proposed by Breiman (1996), and Random Subspace methods. The Bagging method generates several new training sets of size $\boldsymbol{n}$ from the input training set of size $\boldsymbol{N}$, where $\boldsymbol{n}<\boldsymbol{N}$. The Random Subspace method performs a subsampling in the feature space and at each node a set of $\boldsymbol{m}$ features is selected from the input $\boldsymbol{M}$ features, where $\boldsymbol{m}<\boldsymbol{M}$. Each tree in the forest consists of one root node, split nodes and leaf nodes. The root node is the more general node which contains all the input samples. In the split node a split criterion is defined considering one feature $(\varphi)$ and a decision rule $(\tau)$ applied to the feature considered. After the split process all the points at a specific leaf node should belong to a specific class.

At each split node a feature is selected taking into account the importance of the features to the split process (Gao, 2008). There are several possibilities of metrics to assess the importance of attributes (Friedl \& Brodley, 1997). According to Gao (2008), the most common are entropy, information gain and information gain ratio. After defining the application of the features at each split node, the next important consideration is to design an appropriate division rule, through which input data is partitioned into increasingly homogeneous subsets. This is based on the principle of homogeneity of data. It is expected that, with the growth of the tree, the data will be subdivided into homogeneous subsets. The decision rule chosen in a given split node should therefore be the one that provides the greatest homogeneity in the subsets of the descendant nodes. Many algorithms have been developed to establish the division rule in the internal nodes during the growth of a decision tree. Of these rules, the four most common are the Gini index, entropy, the twoing index and probability of class (Zambon et al., 2006).

The RF classifier is implemented and made available by several libraries, one of them in the Python machine learning library Scikit-Learn (Pedregosa et al., 2011) which was used in this work. This library allows the user to set up, among other things: the metrics for measuring the importance of features, the decision rule, and setting the depth and number of trees in the forest. In this work, the entropy metric was used for both feature selection and decision rule definition. Additionally, a depth of 20 and 200 trees was set in the forest. The parameters for depth and number of trees were selected empirically after several tests. The depth varied from 5 to 100 , whereas the number of trees varied from 50 to 10000 .

\section{EXPERIMENTS AND RESULTS}

\subsection{Dataset description}

The dataset corresponds to PPC generated from one aerial survey carried out in the Espigão district of Regente Feijó/Brazil, in the year 2018. The test site (Figure 2) presents high complexity due to densely constructed areas, being composed of buildings of different sizes and shapes located near to each other, isolated trees, clumps of vegetation and power lines. The highlighted regions (R1, R2 and R3) in Figure 2 were selected to visualize the results (Section 3.3).

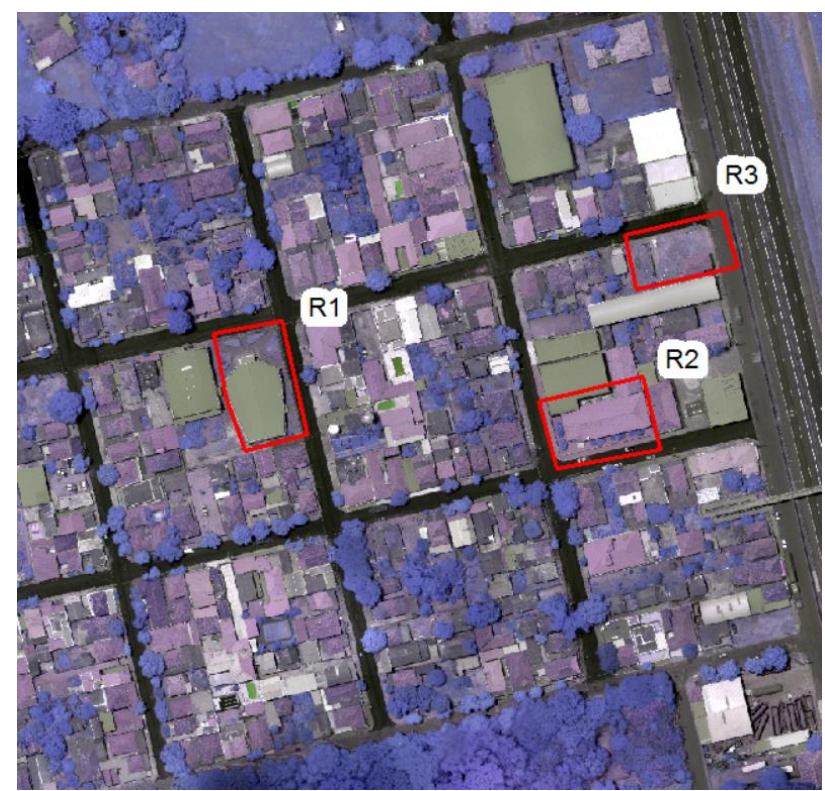

Figure 2. Test site and selected regions (R1, R2 and R3).

The aerial survey was performed using a UAV Sensefly eBee equipped with a positioning system based on GNSS receivers and Micro Electro Mechanical Systems (MEMS) inertial systems. In addition, two dual frequency GNSS receivers were used to obtain 3D coordinates of Ground Control Points (GCP) and check points.

The flight plan parameters are presented in Table 1. A Canon S110 NIR (Near Infra-Red) digital camera with a focal length of $4.40 \mathrm{~mm}$ was used to acquire the images. This camera presents the sensitivity curves of the Green, Red and NIR bands, with central wavelengths positioned at wavelengths $550 \mathrm{~nm}, 625 \mathrm{~nm}$ and $850 \mathrm{~nm}$, respectively.

\begin{tabular}{l|l}
\hline Parameter & Set values \\
\hline Flight height & $120(\mathrm{~m})$ \\
\hline Ground Sample Distance (GSD) & $4.0(\mathrm{~cm})$ \\
\hline Along-track overlap & $80(\%)$ \\
\hline Cross-track overlap & $70(\%)$ \\
\hline
\end{tabular}

Table 1. Flight parameters. 
The photogrammetric processing was carried out using Pix4D Mapper Pro software, employing the Structure from Motion (SfM) algorithm, which consists of the measurement of photogrammetric points of interest, bundle block adjustment and dense image matching for point cloud generation. In total, eight (8) ground control points (GCP) and ten (10) check points were used in the photogrammetric processing to guarantee and assess the accuracy of the results. The point cloud accuracy analyses based on the check points reach RMSE values of $0.10 \mathrm{~m}$ in planimetry and $0.12 \mathrm{~m}$ in altimetry.

Following the Photogrammetric processing, a set of 3D coordinates and $n$ radiometric information is associated with each point of the clouds, where $n$ is the number of bands of the imaging sensor used to acquire the images. The PPC generated contains a total of 3820797 points and presents an average density of 46.24 points $/ \mathrm{m}^{2}$.

\subsection{Pre-processing and feature extraction results}

The experiments were performed over the PPC resulting from the processing of Section 3.1. The pre-processing step results in the DTM and nDSM. The rapidlasso LAStools ground filtering tool enables the setting of parameters related to the relief and to the objects present in the scene. In this work the standard configuration parameter "town or flats" was used considering that the test site presents a soft relief and is composed of small buildings. The DTM extraction results was used as input data for the nDSM estimation.

After the pre-processing step, the radiometric and geometric features were extracted considering the input data (3D coordinate and R, G, NIR information). The feature extraction was aimed at the generation of information complementary to the information already presented in the PPC. Such complementary information constitutes alternatives which assists in the decision making of the classifier. The radiometric and geometric attributes extracted are presented in Figures 3 and 4 respectively.

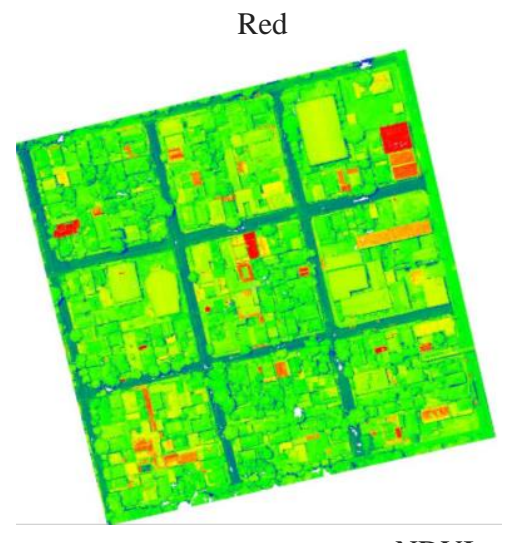

NDVI

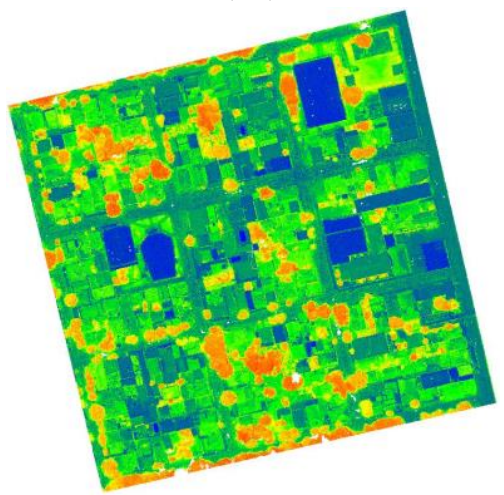

Green

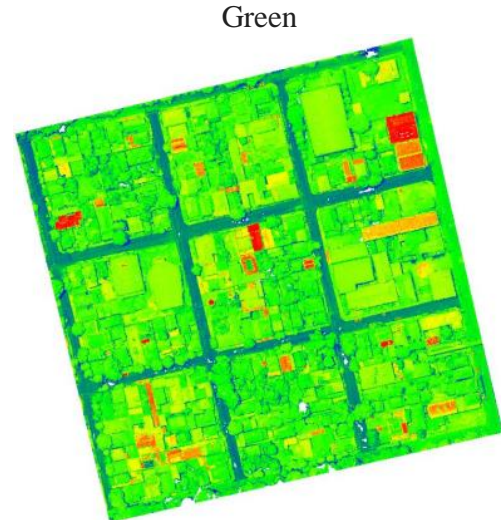

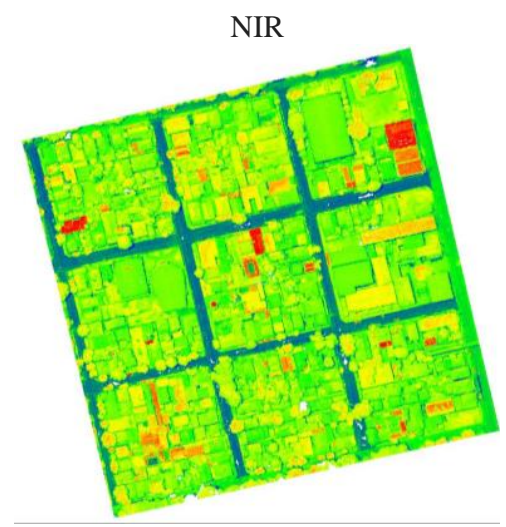

minimum (R, G, NIR)

Figure 3. Radiometric features estimated for the generated PPC. 

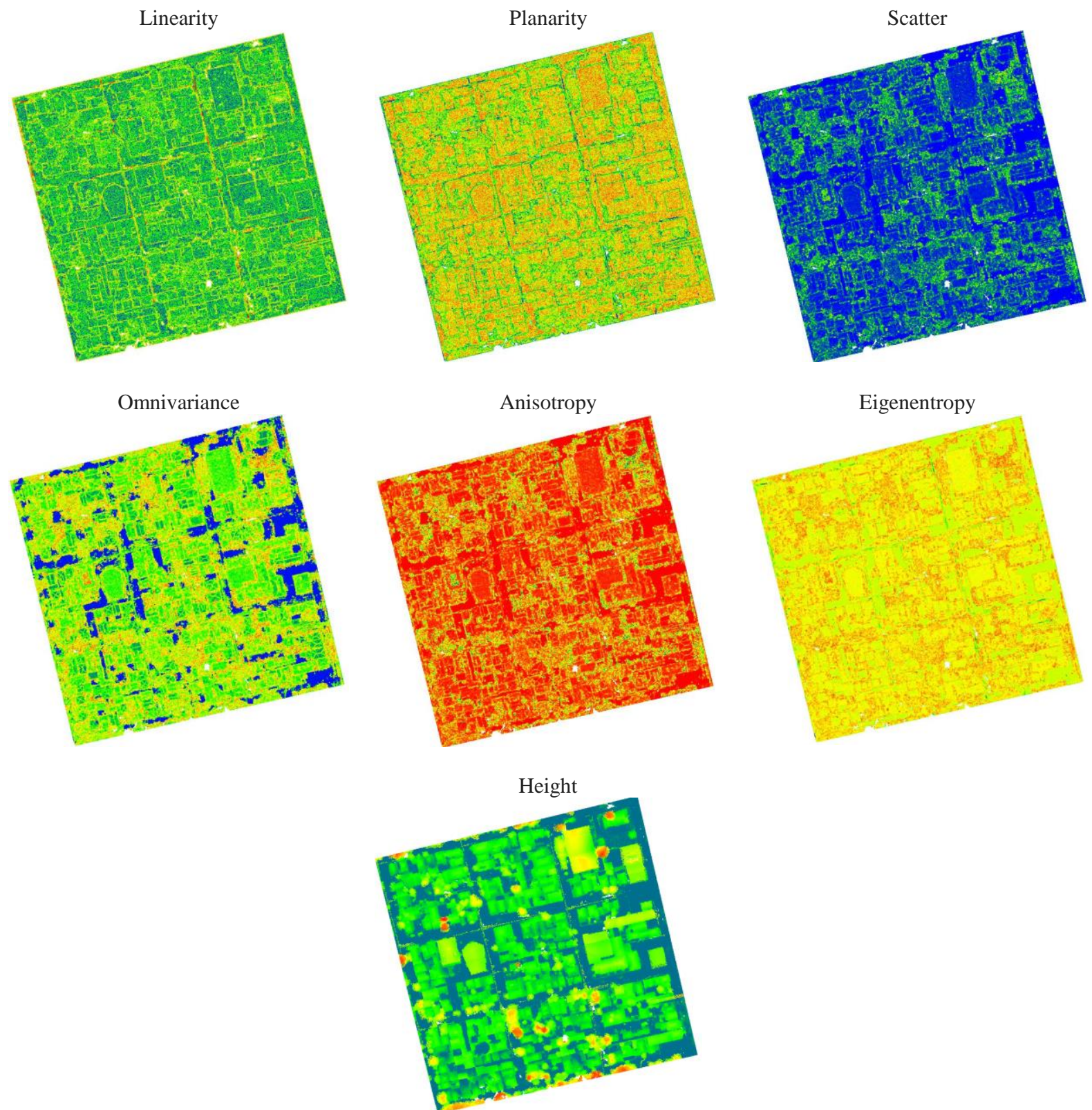

Figure 4. Geometric features estimated for the PPC.

\subsection{Classifier training and application}

Three experiments were carried out considering the extracted features in order to classify the points from the PPC into seven proposed classes: buildings, asphalt, trees, grass, bare soil, sidewalks and power lines. The first experiment was performed using only the radiometric features (Red band, Green band, NIR band, NDVI and minimum (R, G, NIR)). The second considered the use of only the geometric features extracted (Height, Linearity, Planarity, Scatter, Omnivariance, Anisotropy, Eigenentropy). In the last, both the radiometric and geometric features were used.

The classifier training was carried out considering a point cloud sample manually acquired directly over the photogrammetric point cloud by means of visual interpretation using the CloudCompare software. Table 2 presents the sample total number of points per class. From this set of points $60 \%$ were randomly chosen to train the RF classifier and $40 \%$ were used to validate and generate the quantitative analysis.

\begin{tabular}{l|c|c|c}
\hline \multirow{2}{*}{\multicolumn{1}{c|}{ Class }} & \multicolumn{3}{|c}{ Number of points } \\
\cline { 2 - 4 } & Training & Validation & Total \\
\hline Asphalt & 5030 & 3354 & 8384 \\
\hline Bare soil & 5075 & 3383 & 8458 \\
\hline Buildings & 6162 & 4108 & 10270 \\
\hline Grass & 4770 & 3180 & 7950 \\
\hline Side walks & 3367 & 2244 & 5611 \\
\hline Trees & 8446 & 5630 & 14076 \\
\hline Power lines & 10594 & 7063 & 17657 \\
\hline Total & $\mathbf{4 3 4 4 4}$ & $\mathbf{2 8 9 6 2}$ & $\mathbf{7 2 4 0 6}$ \\
\hline
\end{tabular}

Table 2. Total number of points per class, part used for the training and part used for the validation.

The trained classifiers (RF) were applied in order to classify the PPC. The classification results are presented in Figure 5, for the three regions of the test site (highlighted in Figure 2). 
The classification accuracy was evaluated based on the confusion matrix results, from which were derived the recall, precision and overall accuracy for each class, as expressed in $\mathrm{Eq}(\mathrm{s})$. 9-11. Table 3 presents the results of the classification accuracy evaluation for the three experiments performed.

$$
\begin{gathered}
\text { recall }=\frac{t p}{t p+f p} \\
\text { precision }=\frac{t p}{t p+f n}
\end{gathered}
$$

$$
\text { overall accuracy }=\frac{t p+t n}{t p+f p+t n+f n}
$$

where $t p, f p$, tn and $f n$ represent the true positive, false positive,

\begin{tabular}{|c|c|c|c|c|c|c|}
\hline \multirow[t]{2}{*}{ Classes } & \multicolumn{2}{|c|}{ Geometric features } & \multicolumn{2}{|c|}{ Radiometric features } & \multicolumn{2}{|c|}{$\begin{array}{c}\text { Geometric and } \\
\text { Radiometric }\end{array}$} \\
\hline & Recall & Precision & Recall & Precision & Recall & Precision \\
\hline Asphalt & 0.35 & 0.42 & 0.70 & 0.51 & 0.99 & 1.00 \\
\hline Bare soil & 0.70 & 0.55 & 0.90 & 0.90 & 0.94 & 0.93 \\
\hline Buildings & 0.89 & 0.91 & 0.81 & 0.87 & 0.99 & 1.00 \\
\hline Grass & 0.65 & 0.65 & 0.83 & 0.82 & 0.98 & 0.98 \\
\hline Side walk & 0.26 & 0.31 & 0.86 & 0.81 & 0.92 & 0.94 \\
\hline Trees & 0.89 & 0.91 & 0.92 & 0.91 & 1.00 & 1.00 \\
\hline \multirow[t]{2}{*}{ Power lines } & 0.91 & 0.88 & 0.68 & 0.81 & 1.00 & 1.00 \\
\hline & $\begin{array}{c}\text { Overall } \\
\text { accuracy }\end{array}$ & 0.74 & $\begin{array}{c}\text { Overall } \\
\text { accuracy }\end{array}$ & 0.80 & $\begin{array}{c}\text { Overall } \\
\text { accuracy }\end{array}$ & 0.98 \\
\hline
\end{tabular}
true negative and false negative in the confusion matrix respectively.
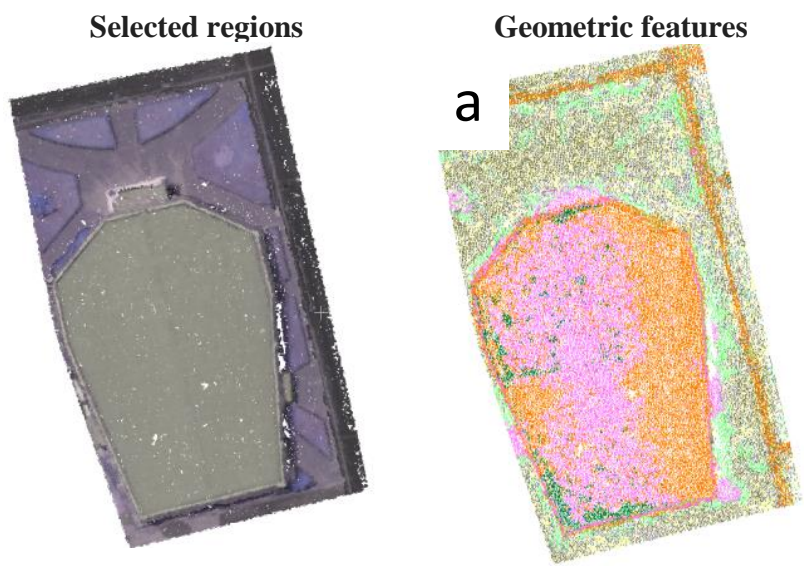

Radiometric features
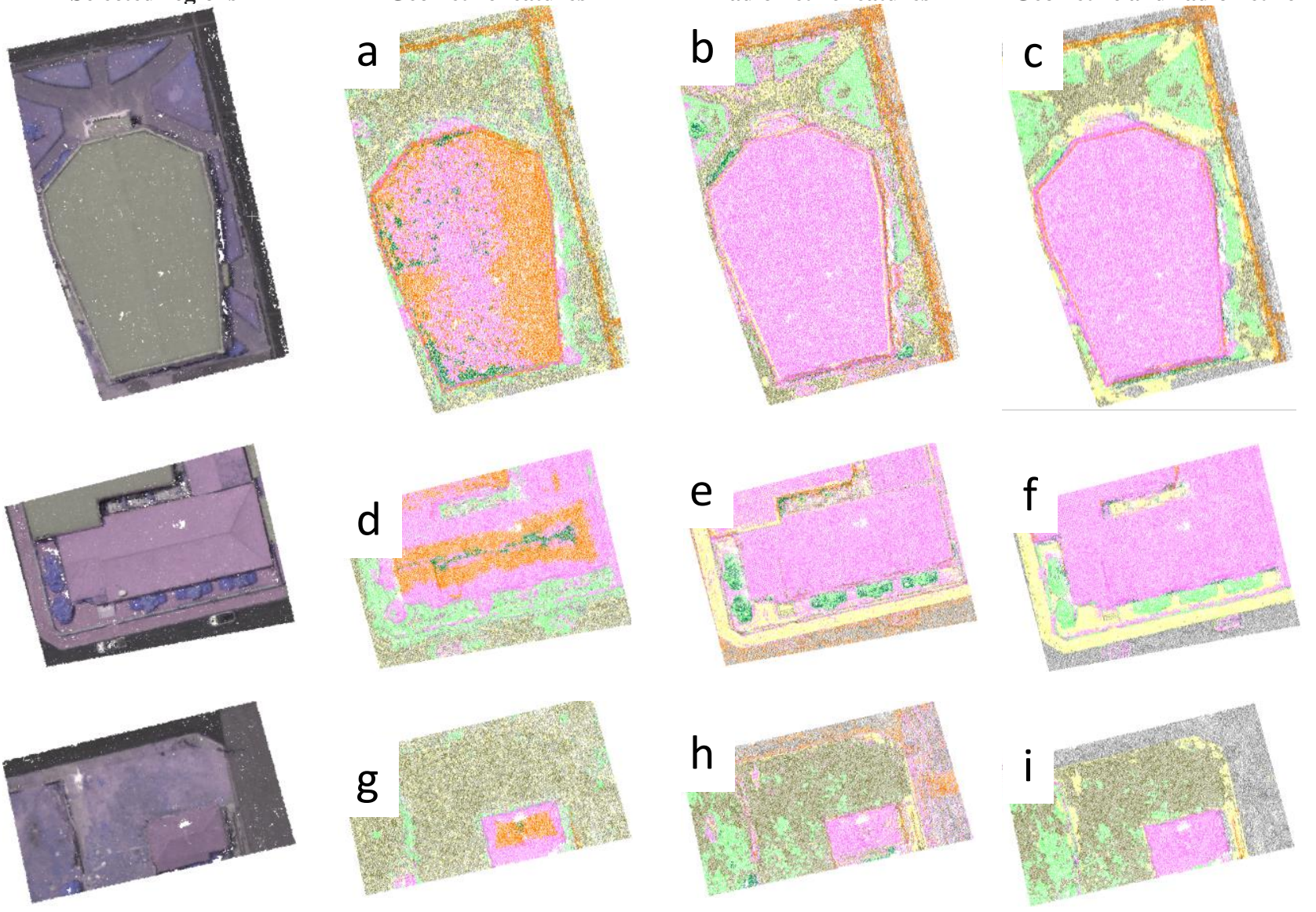

Geometric and radiometric
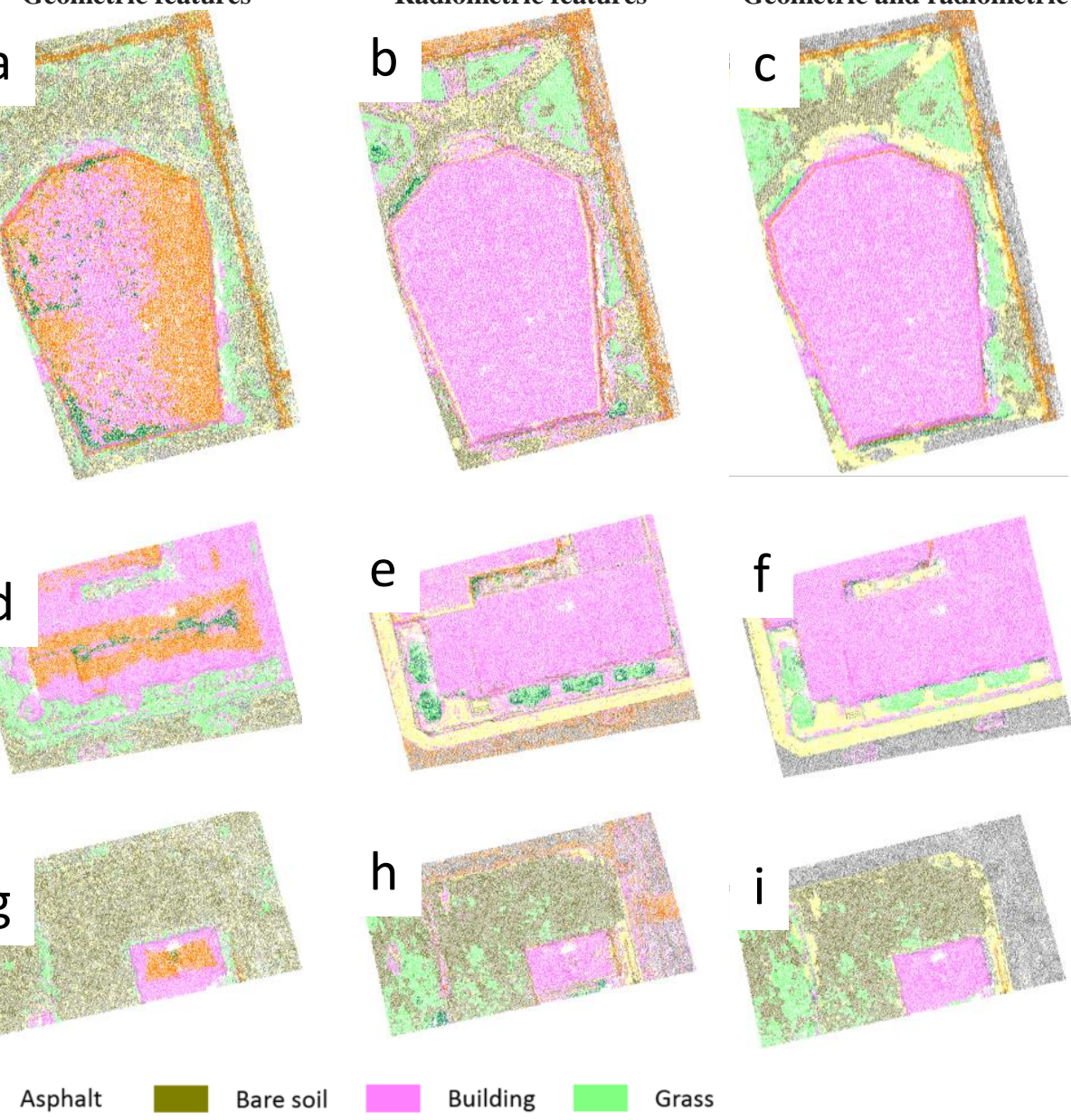

Building

Grass

Power line

Figure 5. Classification results for the three selected regions considering different features. 


\section{DISCUSSION OF RESULTS}

Many features can be considered to assist with urban classification tasks. Some of these were selected and their influence on enabling the classifier to differentiate between objects present in a scene was tested. A visual analysis of the features extracted reveals that the radiometric features Red and Green and the minimum (R, G, NIR) are very similar, and some classes are confused with each other, such as grass and trees, sidewalks and bare soil. However, these features provide the differentiation between the asphalt class and other classes. NIR and especially NDVI features contribute to differentiating vegetation classes (trees and grass) from other classes. By taking account of height, a geometric feature, the differentiation between object classes (buildings, trees and power lines) and ground classes (grass, asphalt, side walk and bare soil) can be noted. In addition, it is possible to observe in Figure 4 that all the features, beside the height feature, present high local variability (roughness) that can be related to both, the PPC generation and the geometric feature extraction, that is affected by the neighborhood.

From a visual analysis of the classification results presented in Figure 6 it can be seen that the classification considering only geometric features (Figure $6 \mathrm{a}, \mathrm{d}$ and g) presents the worst result. This classification was capable of differentiating the object into two more general classes, ground (bare soil, grass, sidewalks and asphalt) and elevated objects (trees, buildings and power lines), but with a high degree of confusion among between them. The high confusion among these elements is directly related to the high local variability of the geometric features. The classification considering only the radiometric features (Figure $6 \mathrm{~b}$, e and h) presents better results than the results from geometric classification, but some confusion still persists, especially in spectrally similar classes such as trees, grass and buildings, asphalt and power lines. However, the results presented by the classification considering both radiometric and geometric features (Figure $6 \mathrm{c}$, f, and i) gave the best results and were capable of differentiating between all the predicted classes.

The quantitative analysis attested the visual analysis discussion. The classification that considered only geometric features yielded the worst values with an overall accuracy of 0.74 . The recall and precision values for the asphalt and sidewalk classes was less than 0.40 , which indicates serious failure to classify these types of object. The values of precision and recall for the bare soil and grass classes ranged from 0.50 to 0.70 , a fact that indicates the classification is not satisfactory. The values for classes that consider objects above ground, such as trees, buildings and power lines, showed values above 0.9 , indicating the capability of these features in the differentiation of the elevated objects. The classification considering radiometric features presented results better than the classification considering geometric features, with an overall accuracy of 0.80 . The recall and accuracy values for the classes considered were above 0.80 , excluding the asphalt and power line classes. The confusion between asphalt and power lines can be seen in Figure $6 \mathrm{~b}$. The classification considering both radiometric and geometric features gave the best results with an overall accuracy of 0.98. The recall and precision values for the classes considered were over than 0.92 .

\section{CONCLUSIONS}

This paper was motivated by the recent development of platforms such as UAV, positioning and inertial sensors and photogrammetric software enabling the generation of high dense photogrammetric point cloud. In this context, this work aimed to explore the potential of radiometric and geometric features extracted directly over PPC considering the Random Forest classifier. This work explores the potential of radiometric and geometric features, isolated and combined, in classification tasks without the need to integrate two different data sources.

Although the visual analysis of the extracted features pointed out the high local variability of geometric features, which implies the potential of these attributes to assist the classifier, the application of these features into machine learning algorithms enables deeper analysis and urban scene classification. Visual and quantitative analysis indicates the potential of radiometric and geometric features extracted directly over PPC for classification tasks, presenting satisfactory results for urban areas with a high level of complexity. In addition, the classification limitations encountered when considering only radiometry or just geometry features were overcome by applying the two feature sets together. The combined use of radiometric and geometric features presented significant improvements when compared to the use only of geometric or radiometric features. As future work, the evaluation of correlations between features, consideration of different parameters in the classifier algorithm and inclusion of a refinement step in the method aiming to reclassify isolated points based in their neighborhood, is suggested.

\section{ACKNOWLEDGEMENTS}

The authors would like to thank the Coordination for the Improvement of Higher Education Personnel - CAPES, for the grant to the first author (Process 1481349); the São Paulo Research Foundation - FAPESP (2014/01841-1 and 2016/12167-5); and National Council for Scientific and Technological Development - CNPq (Process 304189/2016-2) for supporting this research.

\section{REFERENCES}

Aasen, H., Burkart, A., Bolten, A., and Bareth, G., 2015. Generating 3D hyperspectral information with lightweight UAV snapshot cameras for vegetation monitoring: From camera calibration to quality assurance. ISPRS Journal of Photogrammetry and Remote Sensing, 108, 245-259.

Ali-Sisto, D., and Packalen P., 2017. Forest change detection by using point clouds from dense image matching together with a LiDAR-derived terrain model. IEEE Journal of Selected Topics in Applied Earth Observations and Remote Sensing, 10(3), 1197-1206.

Axelsson, P., 1999. Processing of laser scanner dataalgorithms and applications. ISPRS Journal of Photogrammetry and Remote Sensing, 54(2-3), 138-147. doi.org/10.1016/S09242716(99)00008-8.

Axelsson, P., 2000. DEM generation from laser scanner data using adaptive TIN models. International Archives of Photogrammetry and Remote Sensing, 33(4), 110-117. 
Blomley, R., Weinmann, M., Leitloff, J. and Jutzi, B., 2014. Shape distribution features for point cloud analysis - a geometric histogram approach on multiple scales. ISPRS Annals of the Photogrammetry, Remote Sensing and Spatial Information Science, Volume II-3, 9-16.

Breiman, L., 1996. Some properties of splitting criteria. Machine Learning, 24(1), 41-47.

Breiman, L., 2001. Random forests. Machine learning, 45(1), $5-32$.

Buján, S., González-Ferreiro, E., Reyes-Bueno, F., Barreiro-Fernández, L., Crecente, R., and Miranda, D., 2012. Land use classification from LiDAR data and ortho-images in a rural area. The Photogrammetric Record, 27(140), 401-422.

Colomina, I., and Molina, P., 2014. Unmanned aerial systems for photogrammetry and remote sensing: A review. ISPRS Journal of Photogrammetry and Remote Sensing, 92, 79-97.

Demantke, J., Mallet, C., David, N., and Vallet, B., 2011. Dimensionality based scale selection in 3D lidar point clouds. International Archives of Photogrammetry, Remote Sensing and Spatial Information Science. 38(5), 97-102.

Friedl, M. A., and Brodley, C. E., 1997. Decision tree classification of land cover from remotely sensed data. Remote sensing of environment, 61(3), 399-409.

Gao, J., 2008. Digital analysis of remotely sensed imagery. McGraw-Hill Professional.

Haala, N., and Kada, M., 2010. An update on automatic 3D building reconstruction. ISPRS Journal of Photogrammetry and Remote Sensing. 65, 570-580.

Han, X. F., Jin, J. S., Wang, M. J., Jiang, W., Gao, L., Xiao, and L., 2017. A review of algorithms for filtering the 3D point cloud. Signal Processing: Image Communication, 57, 103-112.

Hermosilla, T., Ruiz, L. A., Recio, J. A., and Estornell, J., 2011. Evaluation of automatic building approaches combining high resolution images and LiDAR data. Remote Sensing, 3(6), $1188-1210$.

Li, Z., Zhu, Q., and Gold, C., 2004. Digital terrain modeling: principles and methodology. CRC press.

Malpica, J. A., Alonso, M. C., Papí, F., Arozarena, A., and Martínez De Agirre, A., 2013. Change detection of buildings from satellite imagery and LiDAR data. International Journal of Remote Sensing, 34(5), 1652-1675.

Meyer-Baese, A., and Schmid, V. J., 2014. Pattern recognition and signal analysis in medical imaging. Elsevier.

Näsi, R., Honkavaara, E., Blomqvist, M., LyytikäinenSaarenmaa, P., Hakala, T., Viljanen, N., Kantola, T., and Holopainen, M., 2018. Remote sensing of bark beetle damage in urban forests at individual tree level using a novel hyperspectral camera from UAV and aircraft. Urban Forestry \& Urban Greening, 30, 72-83.

Nex, F., and Remondino, F., 2014. UAV for 3D mapping applications: a review. Applied geomatics, 6(1), 1-15.
Pedregosa, F., Varoquaux, G., Gramfort, A., Michel, V., Thirion, B., Grisel, O., Blondel, M., Prettenhofer, P., Weiss, R., Dubourg, V., Vanderplas. J., Passos, A., Cournapeau, D., Brucher, M., Perrot, M., and Duchesnay, É. 2011. Scikit-learn: Machine learning in Python. Journal of machine learning research, 12, 2825-2830.

Pfeifer, N., Köstli, A., and Kraus, K., 1998. Interpolation and filtering of laser scanner data-implementation and first results. International Archives of Photogrammetry and Remote Sensing, 32, 153-159.

Rau, J. Y., Jhan, J. P., and Hsu, Y. C., 2015. Analysis of oblique aerial images for land cover and point cloud classification in an urban environment. IEEE Transactions on Geoscience and Remote Sensing, 53(3), 1304-1319.

Santos, R. C., Galo, M., and Carrilho, A. C, 2019. Extraction of building roof boundaries from LiDAR data using an adaptive alpha shape algorithm. IEEE Geoscience and Remote Sensing Letter. doi.org/ 10.1109/LGRS.2019.2894098.

Sithole, G., and Vosselman, G., 2001. Filtering of laser altimetry data using a slope adaptive filter. International Archives of Photogrammetry Remote Sensing and Spatial Information Sciences, 34(3), 203-210.

Sohn, G., and Dowman, I., 2007. Data fusion of high-resolution satellite imagery and LiDAR data for automatic building extraction. ISPRS Journal of Photogrammetry and Remote Sensing, (68), 43-63.

Weinmann, M., Jutzi, B., and Mallet, C., 2013. Feature relevance assessment for the semantic interpretation of 3D point cloud data. ISPRS Annals of the Photogrammetry, Remote Sensing and Spatial Information Science, Volume II-5/W2, 313-318.

Weinmann, M., Jutzi, B., Hinz, S., and Mallet, C., 2015. Semantic point cloud interpretation based on optimal neighborhoods, relevant features and efficient classifiers. ISPRS Journal of Photogrammetry and Remote Sensing. 105, 286304.

Zambon, M., Lawrence, R., Bunn, A., and Powell, S., 2006. Effect of alternative splitting rules on image processing using classification tree analysis. Photogrammetric Engineering \& Remote Sensing, 72(1), 25-30.

Zhang, J., and Lin, X., 2013. Filtering airborne LiDAR data by embedding smoothness-constrained segmentation in progressive TIN densification. ISPRS Journal of Photogrammetry and Remote Sensing, 81, 44-59.

Zhang, J., and Lin, X., 2017. Advances in fusion of optical imagery and LiDAR point cloud applied to photogrammetry and remote sensing. International Journal of Image and Data Fusion, 8(1), 1-31. 